\title{
A Description-Experience Gap in Social Interactions: Information about Interdependence and Its Effects on Cooperation
}

\author{
JOLIE M. MARTIN ${ }^{1 *}$, CLEOTILDE GONZALEZ ${ }^{1}$, ION JUVINA ${ }^{2}$ and CHRISTIAN LEBIERE ${ }^{3}$ \\ ${ }^{1}$ Social and Decision Sciences, Carnegie Mellon University, Pittsburgh, PA, USA \\ ${ }^{2}$ Psychology, Wright State University, Dayton, $\mathrm{OH}, \mathrm{USA}$ \\ ${ }^{3}$ Psychology, Carnegie Mellon University, Pittsburgh, PA, USA
}

\begin{abstract}
In social interactions, decision makers are often unaware of their interdependence with others, precluding the realization of shared long-term benefits. In an experiment, pairs of participants played an Iterated Prisoner's Dilemma under various conditions involving differing levels of interdependence information. Each pair was assigned to one of four conditions: "No-Info" players saw their own actions and outcomes, but were not told that they interacted with another person; "Min-Info" players knew they interacted with another person but still without seeing the other's actions or outcomes; "Mid-Info" players discovered the other's actions and outcomes as they were revealed over time; and "Max-Info" players were also shown a complete payoff matrix mapping actions to outcomes from the outset and throughout the game. With higher levels of interdependence information, we found increased individual cooperation and mutual cooperation, driven by increased reciprocating cooperation (in response to a counterpart's cooperation). Furthermore, joint performance and satisfaction were higher for pairs with more information. We discuss how awareness of interdependence may encourage cooperative behavior in real-world interactions. Copyright (C) 2013 John Wiley \& Sons, Ltd.
\end{abstract}

KEY WORDS cooperation; game theory; interdependence; prisoner's dilemma; pro-social behavior; punishment; reciprocity; social interaction

\section{INTRODUCTION}

In modern society, the welfare of individuals is increasingly dependent on the actions of others. We live in closer contact, work in larger organizations, and rely on expanding public infrastructures. In nearly every aspect of daily life, we influence others through our choices and the choices of others affect us. For example, when people litters in a public place, the presence of trash may cause others to treat the shared space with equal disrespect, resulting in an escalating negative impact on one another. Positive behaviors are equally self-perpetuating: when others respect common resources, we are more likely to do the same, even if we do not explicitly realize the source of influence (e.g., Cialdini, Reno, \& Kallgren, 1990). Although members of a common social system have highly correlated satisfaction levels (Kelley \& Thibaut, 1978), we do not often recognize our mutual accountability for one another's well-being. If we inaccurately assess how our own actions contribute to our own outcomes and the outcomes of others, it is difficult to discover tradeoffs that maximize joint welfare or even individual welfare. In this way, information about others' behaviors and preferences affects the degree to which we are able to improve our decisions from either an individual or societal standpoint.

Ambiguity about our interdependence with others is pervasive in many types of relationships ranging from corporate negotiations to romantic partnerships (Rusbult \& Van Lange, 2003). The unfulfilled desire of a frustrated businessman or lovelorn soul to understand precisely what his

*Correspondence to: Jolie M. Martin, Social and Decision Sciences, Carnegie Mellon University, Pittsburgh, PA, USA. E-mail: joliemm@gmail.com partner is thinking and feeling is testament to this. At the most basic level, the very existence of other people as actors in our social sphere may be unknown. In the stock market, for instance, it is unclear to what extent a large jump or drop in the public valuation of a security is due to a direct result of deliberate human action versus a by-product of computerized algorithms (albeit programmed by humans). Furthermore, even if individuals are identifiable by name or alias, their actions and outcome preferences may not be transparent. In open source projects like Wikipedia, for example, behind-the-scenes contributors are ad hoc networks of anonymous individuals who rarely communicate one-on-one, much less face-to-face (Moore, Kurtzberg, Thompson, \& Morris, 1999). When something gets done well or a mistake is made, the average user does not know who is accountable. This is especially true in organizations where collaborators from different functional areas have disparate-and possibly incompatible-objectives that cannot be correctly inferred from one's own. Finally, and perhaps most importantly, observable behaviors may offer little insight about the underlying motivations of others. In complex interactions, as interdependent decision makers react to one another over time, personal and situational effects on others cannot be easily isolated to understand why any one person acted as he or she did or to predict how he or she will behave in the future. For example, as a commuter in a high-traffic urban area, you would probably be aware that each driver puts stress on the overall system, but you would not know the specific alternatives (e.g., public transportation) available to each driver or the costs and benefits that he incurs for exercising these alternatives.

Although complete information is a common assumption in empirical game theory paradigms, these idealized hypothetical 
scenarios do not adequately reflect the types of uncertainty that people face in real-world social interactions. In reality, decision makers usually understand their interdependence with others only through experience, by the observation of actions and outcomes in repeated interpersonal encounters. Here, we directly test how behavior differs in social interactions based on the amount of descriptive information that decision makers have at their disposal regarding the actions and payoffs of others.

Extrapolating from research on risk-taking in individual choice, we expect to see differences in cooperative behavior, which is one type of social risk, between interactions including descriptive social information compared with those informed by individual experience alone. One prominent finding from this literature is a "description-experience gap" indicating that individual risky choice depends on how information is presented (Hertwig, Barron, Weber, \& Erev, 2004): whether decision makers are given explicit descriptions of outcomes and their associated probabilities (decisions from description (DFD)), or whether decision makers rely upon their own experience to discover outcomes and their likelihoods (decisions from experience (DFE)). The most robust result from this research is that, in contrast to those with descriptive information, people relying on experience alone behave as if they underweight the likelihood of rare outcomes compared with their objectively defined probability of occurrence (Barron \& Erev, 2003; Hertwig et al., 2004; Yechiam, Barron, \& Erev, 2005). Our goal in the current research is to determine whether a similar "information gap" exists in social interactions by studying the effects on cooperation of various amounts of descriptive information - in addition to experiential information - in a well known social dilemma, the Iterated Prisoner's Dilemma (IPD).

\section{THE VALUE OF INFORMATION IN SOCIAL DILEMMAS}

We investigate how information about interdependence with others affects cooperation and the realization of mutually beneficial outcomes in the IPD. The IPD is frequently used in game theory research to study the interplay between concurrent collaborative and adversarial interdependencies. In each round of the IPD, two players decide without communication whether to cooperate or defect, with defection leading to higher outcomes for each regardless of the other's action but mutual cooperation leading to higher joint outcomes than mutual or unilateral defection (e.g., payoffs as in Table 1). Across multiple rounds of interaction (infinitely repeated in theory; finitely repeated with unknown endpoint in practice), players face a tradeoff between the guaranteed short-term gain of defection and the potential long-term gain of cooperation that is only realized if the other player is influenced to cooperate, too.

Given the multiplicity of equilibria that can be supported in the IPD (Roth \& Murnighan, 1978), information is especially important for decision makers to infer one another's
Table 1. Prisoner's dilemma payoff matrix, with Action A denoting defection and Action B denoting cooperation

\begin{tabular}{lccc}
\hline & \multicolumn{2}{c}{ Player 2 Action } \\
\cline { 3 - 4 } & & $\mathrm{A}$ & $\mathrm{B}$ \\
\hline Player 1 Action & $\mathrm{A}$ & $-1,-1$ & $10,-10$ \\
& $\mathrm{~B}$ & $-10,10$ & 1,1 \\
\hline
\end{tabular}

The cells show a pair of outcomes $(x, y)$ where $x$ is the payoff to Player 1 and $y$ is the payoff to Player 2 .

intentions and to predict one another's actions. For example, Rapoport and Chammah (1965) demonstrated that participants who viewed a payoff matrix cooperated more than those who learned payoffs through experience alone ( $46 \%$ vs. $22 \%$ cooperation). The present experiment expands upon these findings by exploring how players' behavior changes across a broader spectrum of information conditions, including those with little to no information about the nature of interdependencies with another player, and by looking at the trends in cooperation over time. In general, the more information one obtains about others' actions and outcomes during the course of interaction, the more accurately one will be able to infer their true motivations by extrapolating the interdependencies in the payoff matrix (Blount, 1995; Nisbett \& Ross, 1980). While this may seem self-evident, it is not obvious whether this will lead to more or less cooperation: each player may indeed seek to maximize his own gains, and only a certain subset of reasonable assumptions by each player about the other leads to sustained cooperation as a best response strategy, even when full information is available. However, we argue that explicit information about others' actions and outcomes will increase cooperation between two individuals interacting in the IPD because of the particular assumptions that people tend to make in response to varying amounts of descriptive information. Specifically, we expect that more information will increase the perceived likelihood that a counterpart will cooperate, as well as increase the perceived benefit via reciprocity of taking the cooperative action oneself.

Although the value of certain types of information has already been highlighted in well known social games such as the IPD (Rapoport \& Chammah, 1965; Camerer 2003), it is unclear how more information systematically influences the emergence of cooperation. A common but unrealistic assumption in research on strategic social interaction is that individuals possess full information about their interdependence, represented in the IPD by a matrix that specifies how joint actions map to joint outcomes (e.g., "if you do A and I do B, you will get X and I will yet Y") (Camerer \& Fehr, 2006). We examine repeated risky choices in the IPD, but in contrast to most studies, we manipulate the two players' information about their interdependency across four experimental conditions. The most ambiguous condition is a direct link to individual decisions from experience in binary choice tasks, where decision makers are completely unaware of their interaction with another player and may perceive variations in their outcomes to result from stochastic 
factors. We incrementally increase the amount of information available to players in different conditions of the IPD until reaching the typical experimental paradigm in behavioral game theory where full descriptive information about the outcomes associated with joint actions is provided from the outset of the interaction in the form of a payoff matrix.

The levels of information we use are based on the Hierarchy of Social Information (HSI) (Gonzalez \& Martin, 2011), a theoretical framework for conceptualizing the major categories of interpersonal information that may play a role in social interactions as shown in Figure 1. We will summarize each of these informational layers (with the exception of the top layer on "Cultural" information, which is beyond the scope of this paper) and provide the real-world intuition for how additional information at each level could promote cooperation in social interactions before offering more theoretical evidence in the subsequent section.

At the lowest "No-Info" information level of the HSI (which Gonzalez \& Martin, 2011, refer to as Non-Social), actors are indeed interdependent (based on the IPD structure in which both players' actions determine each of their payoffs), but they do not have any knowledge of this fact. People only observe their own actions and outcomes without realizing that they influence or are influenced by others. Lacking awareness of interdependence, decision makers are apt to attribute variability in their own outcomes to random processes or deterministic patterns rather than to the agency of another human being. From each individual's perspective, we expect that the choice context will appear very similar to individual DFE (Hertwig et al., 2004) where players interact with "nature" (probability distributions). For example, a writer who enjoys working at a local café might sometimes experience a slow connection on his or her laptop due to other customers streaming large videos, but if he or she is unaware of this cause, he or she might attribute the problem to an occasional technical malfunction that happens to be more persistent on some days than others. If so, the amount of available bandwidth would seem like mere chance or a

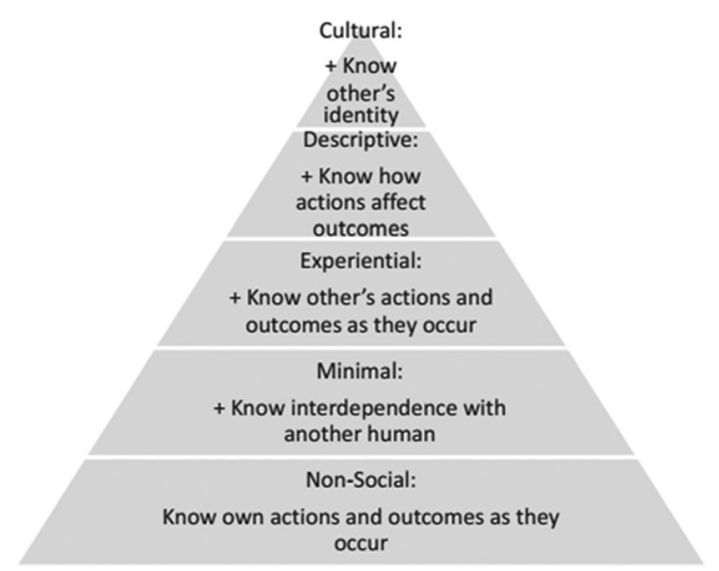

Figure 1. The Hierarchy of Social Information: "Each level of the pyramid represents a category of interaction, with social information compounding from the bottom row (least information) to the top row (most information). Each interaction type includes all of the information below, as well as one new type of information"

(Gonzalez \& Martin, 2011) probability that he or she must weigh in deciding whether to go to the café versus stay at home and use a more reliable connection. On days when he or she is at the café and the connection is particularly strong, he or she might even take advantage of it by watching a few videos, not realizing that he or she is then causing a problem for others. We will treat behavior in this setting as a baseline for comparing behavior in layers of the HSI with incrementally enhanced awareness of interdependence.

Unlike those with no social information, decision makers at the "Min-Info" information level (the Minimal information level in Gonzalez \& Martin, 2011) are aware of their interdependence with another human being. However, they do not know how their own actions influence others, and vice versa. This situation often arises when interacting individuals try to discern others' preferences without seeing their precise actions or outcomes. For instance, a sign at one's work cafeteria may suggest that employees not take too much of any particular food item in order to leave enough for others. While it is true that individuals are interdependent in such situations of limited shared resources, it would be suboptimal if everyone followed the simplest norm, taking small equal amounts of every dish, even though some prefer broccoli and others prefer cake. If left to their own devices, it is imaginable that even employees who went through the food line at different times (and hence could not observe one another's choices or satisfaction with outcomes) would begin to notice which items were more depleted and reach a state in which people held back only on the widely popular items. In the IPD, we expect that this minimal social information may enhance cooperation through (i) a win-stay-lost-shift strategy or (ii) a tit-for-tat strategy. Both cases require decision makers to realize that the "probability" they will receive a particular outcome for a particular action is dynamic because it hinges upon the action taken by the other player. In the first case, if by coincidence, both players adopt a win-staylose-shift approach as a result of this realization of interdependence, they will converge to mutual cooperation regardless of their initial actions: if both cooperate simultaneously (producing a positive outcome-a win), they will persist in that action; if they defect simultaneously (producing a negative outcome-a loss), they will both then switch to the alternate action corresponding to cooperation; if they take asymmetric actions, the player who defected will stay and the player who cooperated will shift, such that they will both lose in the subsequent round and revert to mutual cooperation. They will then persist in playing the cooperative action. In the second case, a very savvy decision maker might adopt a strategy that yields the equivalent actions as tit-for-tat based solely on his or her own outcomes, which allow him or her to infer his or her counterpart's actions and outcomes because of their symmetry to his or her own. Although this player would initially take random actions until he or she discovered the possible set of outcomes, he or she would subsequently play tit-for-tat by following a loss (due to the other defecting) by defecting himself, and by following a gain (due to the other cooperating) by cooperating himself. For this strategy to increase cooperation overall, the other player would at least need to recognize how his 
or her actions were being negatively or positively reinforced, and respond with greater cooperation. While these scenarios of increased cooperation with minimal information are plausible, they seem unlikely because they require a great deal of insight on the part of both players, and of course, accountability remains at a minimum when people cannot directly observe the impact of their actions on the outcomes of others, and vice versa.

The next "Mid-Info" level of the HSI (referred to as Experiential information by Gonzalez \& Martin, 2011) adds information about the actions and outcomes of others as they take place over time, which allows people to learn over time how they depend on one another. This represents a real-world situation such as friends deciding whether to make an effort to arrive to an appointment on time. If one person is late, this is observable to others and each of their costs or benefits (e.g., who has to wait and who does not) are observable, as well. Knowledge of this interdependence is apt to increase the likelihood of all parties arriving on time so that no one has to wait. In general, we expect that the confluence of strategic and affective responses to experiential information about others' actions and outcomes will increase cooperation in "public goods" problems like the IPD, as decision makers recognize that there is potential for mutual gain over the long term.

Continuing up the HSI, the "Max-Info" level of information (called Descriptive information by Gonzalez \& Martin, 2011 ) is one found often in laboratory studies of the IPD, yet encountered relatively rarely in the real world (Camerer $\&$ Fehr, 2006). Descriptive information about the structure of an interaction specifies the contingencies between all parties' actions and outcomes, as shown in a typical game theory payoff matrix. An example of this sort of descriptive information is if two competing companies such as Coke and Pepsi formalized the market share and profits each would receive depending on the price that each set. Clearly defining this range of possibilities would enable decision makers at each company to see that collusion to maintain high prices would be in both of their long-run interests even though they might get a short-term gain by undercutting the other on any given week. This sort of knowledge in social interactions is analogous to summary information about outcome probabilities and payoffs in individual DFD (Hertwig et al., 2004), with the additional complexity that descriptive information is included as an additional layer on top of experiential information (as discussed in Lejarraga \& Gonzalez, 2011). Given the extensive literature on a "description-experience gap" in individual choice, contrasting behavior with and without descriptive information, the relevance to interdependence information in social interaction warrants more detailed attention.

\section{A DESCRIPTION-EXPERIENCE GAP IN SOCIAL INTERACTIONS}

The literature on individual decision making reveals a description-experience gap or difference in risky choice between DFE and DFD contexts. The most robust result from this literature is that, in contrast to predictions of risky choice in DFD, small probabilities appear to be given less weight than their objective values in DFE (Barron \& Erev, 2003; Hertwig et al., 2004; Yechiam et al., 2005). This may be attributable in part to reliance on small samples where rare events are statistically underrepresented (Rakow, Demes, \& Newell, 2008) or to decision makers' false assumptions that low probability events are impossible or that high probability events are certain (Hadar \& Fox, 2009). However, recent studies have ruled out judgment error as a singular explanation for the divergence in risky choice between DFE and DFD (Ungemach, Chater, \& Stewart, 2009), reaffirming that fundamental differences exist in the representation of risk as natural frequencies in DFE but as a priori probabilities in DFD (Hau, Pleskac, \& Hertwig, 2009; Hau, Pleskac, Kiefer, \& Hertwig, 2008).

Although research on the description-experience gap has emphasized distinctions in probability perceptions between DFE and DFD, there is also recent work indicating divergence in the subjective impact of the same objective outcomes across the two paradigms. Specifically, Ludvig and Spetch (2011) conducted an experiment of risky choice in which small and large numerical outcomes were equally likely in both the gain and loss domains, varying only the experiential or descriptive nature of the outcomes. Their results are consistent with the "reflection effect" of greater risk-taking for losses than for gains in DFD, which can be accounted for by diminishing marginal utility over objective outcomes with distance from a zero reference point (Kahneman $\&$ Tversky, 1979). In other words, observed choices are consistent with a convex utility function for losses but a concave utility function for gains. However, participants in that study exhibited greater risk-taking for gains than for losses in DFE, suggesting the opposite pattern of increasing marginal utility (greater impact of extremes) when descriptive information was absent and experience was the sole source of information. In related studies, Yechiam and Busemeyer (2006) found greater choice of risky options with rare, large negative payoffs when information about foregone outcomes was available in addition to experience. Although this could be interpreted to mean that decision makers with additional information gave small probabilities less weight, the authors argue that the divergence from prior findings may be caused by the discounting (and even negative weighting by some participants) of large negative foregone payoffs. More research is needed on this phenomenon, yet these results support the proposition that descriptive information mitigates the effect of extreme positive or negative outcomes on choice, regardless of their probabilities. Conversely, experience alone may accentuate the impact of extreme positive and negative outcomes on subsequent decisions.

The aforementioned studies highlight variations in individual choice with information about one's own outcome probabilities and magnitudes. Several studies have begun to extend this work to the domain of social behavior. For example, Baker and Rachlin (2001) simulated an IPD in which individual participants relied on experience alone or on experience plus description to assess a computerized counterpart's probability of reciprocation. They found that 
the addition of descriptive information increased participants' optimization, as is the case for a larger class of probabilistic individual decision problems (Fantino \& Navarro, 2012). In the present research, we test variations in social behavior in actual two-player interaction in the IPD, which allows for realistic shifts over time in the "probabilities" (i.e., human propensities) that another player will take particular actions. We examine cooperation as interacting decision makers are given more or less information about their interdependence, namely the contingency of each individual's outcomes on one another's actions. Just as the DFE paradigm relaxes assumptions about the descriptive information available in individual decision tasks, we do the same for descriptive information about interdependence with others in social dilemmas. We identify two main analogies between the role of descriptive information in social choice and in individual choice, the first relating to the perceived probabilities of various outcomes and the second to the subjective impact of various outcomes.

The first analogy between the description-experience gap in individual choice and the one we posit for social choice pertains to the way that decision makers assess the likelihood of obtaining various outcomes. By definition, interdependence between two people implies that each of their actions affects the other's outcomes; uncertainty about another person's preferences and proclivities is analogous to the risk of selecting a probabilistic option in individual choice. Like the "frequentist" representation of chance occurrences in individual DFE, a decision maker engaged in social interaction from experience may selectively piece together instances from memory about another's propensity to take particular actions and the likely proportion of contingent outcomes for himself or herself. If the demonstrated contrast between DFE and DFD is any guide (e.g., Ungemach et al., 2009), people will be apt to underweight the probability of rare events when they lack summary information about their interdependence with others. Although either cooperation or defection could be rarer given the game's evolution, the inability to assess game symmetry with lower levels of descriptive information could cause a rush to judgment about the stability of another person's character traits-especially negative attributions - that will presumably persist into the future (Jones \& Harris, 1967; Pillutla \& Chen, 1999). Further up the information spectrum, greater awareness of interdependence could serve as a reminder that another person can alter his or her behavior at any time even if his or her most frequent or recent actions appear consistent. In particular, while the possibility of defection by the other is salient by default, the payoff matrix gives equal visual "real estate" to all four outcomes, offering an additional reminder that the other player has agency to take the cooperative action. This supports our prediction that decision makers with more interdependence information will develop a more accurate perspective of their counterparts' potential actions. Thus, we expect that additional information will have positive effects on cooperation in interactions such as the IPD where hostility can rapidly escalate if negative attributions are erroneously ascribed to one's counterpart.

The second analogy between individual choice and social choice relates to the way that individuals experience outcomes. Just as decision makers discover outcomes over time in individual DFE, they often discover other people's outcomes through repeated feedback in social interaction. This produces not only knowledge about one's own gains or losses, but also about one's performance or status relative to members of a comparison individual or group (Blount, 1995; Festinger, 1954). Extrapolating from differences between DFE and DFD in individual decision making where additional information in the latter appears to reduce the subjective impact of extreme outcomes (Ludvig \& Spetch, 2011), we also expect that descriptive social information will reduce the subjective impact of outcomes that are very good or bad relative to those of an interaction partner. This dampening of the emotional impact felt from extreme relative outcomes would encourage cooperative behavior in interactions such as the IPD, insofar as it allays fears of making gullible concessions that greatly benefit another person at one's own expense or stifles greedy desires to achieve large gains even if they come at the other's expense (Hwang \& Burgers, 1997).

Indeed, there is abundant evidence that fundamental cognitive processing differs as a result of conscious interdependence with another person (McCabe, Houser, Ryan, Smith, \& Trouard, 2001; Gallagher, Jack, Roepstorff, \& Frith, 2002; Rilling, Sanfey, Aronson, Nystrom, \& Cohen, 2004). For example, Rilling et al. (2008) showed that affect-related neurological activation was greater in response to unreciprocated cooperation than in response to unsuccessful risk-taking in non-social contexts. This differential cognitive processing may relate to an enhanced "theory of mind" or efforts to impute the mental states of another person (Premack \& Woodruff, 1978). If decision makers are aware that others will react to their own actions in the IPD setting, they will realize the need to appease and/or coerce others in order to achieve personal gains. Increased knowledge of interdependence at each level of the HSI may also suffice to trigger a sense of self-efficacy due to perceived control over another person (Bandura, 1982) and understanding of others' intentionality that induces concerns about distributive and procedural justice (Blount, 1995). Specifically, a decision maker might care about fairness in the relative outcomes received by himself or herself and others (Rabin, 1993). Over time, he may try to reinforce favorable behaviors in interaction partners by reciprocating cooperation and punishing defection (Clark \& Sefton, 2001). Individuals may also attempt to build trust (Cox, 2004; Hwang \& Burgers, 1997; Juvina, Saleem, Martin, Gonzalez, \& Lebiere, 2013) and establish a reputation that promotes the credibility of both threats of punishing defection and promises of consistent cooperation (Kreps \& Wilson, 1982; Tinsley, O’Connor, \& Sullivan, 2002).

Taken together, comparisons of probability weighting and outcome impact across DFE and DFD, as well as research on the awareness of human interdependence, lead us to the prediction that increasing descriptive information in social settings will be conducive to cooperation. This is not a straightforward logical conclusion, however, as social behavior also depends on people's beliefs about the interaction structure (Halevy, Sagiv, Roccas, \& Bornstein, 2006) and 
the activation of cooperative or competitive relationship norms (Pillutla \& Chen, 1999). One possibility contrasting our own account is that increasing information could accentuate competitive tendencies by attuning decision makers to the impersonal aspects of the strategic context. This would suggest that observing another person's outcomes over time, and further seeing the game structure in matrix form, would heighten the desire to do better in relative terms, even at the expense of improving absolute levels of individual or joint performance. Further, knowledge of another player's outcomes has been shown to increase tendencies to select the option that has the better outcome most of the time (Yechiam, Druyan, \& Ert, 2008), which would discourage individuals from breaking out of a pattern of mutual defection. Our laboratory study aims to explicitly test our account versus these alternate potential effects of increasing descriptive information about an opponent's actions and outcomes.

\section{HYPOTHESIZED EFFECTS OF INCREASING INFOR- MATION IN THE ITERATED PRISONER'S DILEMMA}

The primary hypothesis of our laboratory experiment was that increasing interdependence information would increase average rates of individual and mutual cooperation in the IPD. Based on the parallels to descriptive information in individual risky choice elaborated earlier, we expected that each incremental increase in information would reduce biased attributions about a counterpart's character while also deemphasizing the attainment of extreme gains and avoidance of extreme losses. This more "moderate" perspective would help to prevent people from jumping to conclusions about the inevitability of sustained mutual defection, even if they realized that defection was the more attractive option for increasing personal gain in a single round. Especially in settings like the IPD where the two players' available actions and payoffs are symmetric, more information would illuminate the shared plight of one's counterpart. By inspiring empathy, interdependence information could induce more cooperation partly as an act of generosity toward the other player (Charness \& Rabin, 2002). This cooperation need not be driven by "pure" altruism; however, because the desire to maintain a positive self-image of contribution to the common good might also be triggered by acknowledging one's own responsibility for joint outcomes (Festinger, 1957; Tetlock, 1992).

Moreover, we predicted that players with more interdependence information would engage in higher-level reasoning about how counterparts were likely to interpret their own actions. Additional information would underscore the futility of earning large one-shot gains through defection if it deterred a counterpart from cooperating in the future and, at the same time, the futility of repeated cooperation if one was consistently taken advantage of by a defecting counterpart. This reasoning would cause participants to adhere more closely to a tit-for-tat strategy of repeating a counterpart's most recent action, which is found to be simple and effective in a range of evolutionary settings (Axelrod \& Hamilton,
1981; Axelrod, 1984). If so, incremental information would promote both reciprocation (higher propensity to cooperate, contingent on a counterpart's prior cooperation) and punishment (higher propensity to defect, contingent on a counterpart's prior defection). This pattern would provide evidence that higher rates of cooperation across the course of the game were not acts of blind allegiance to the common good but rather strategic threats or rewards to a counterpart who defected or cooperated, respectively, in the previous round.

Lastly, based on the confluence of cooperative tendencies and the deterrence of exploitative defectors, we predicted that information would increase pair-level performance (the sum of the two players' point totals) and satisfaction (the sum of the two players' rated satisfaction with the game outcome) and that the effect of information on both would be mediated by a pair's level of mutual cooperation. This would indicate that additional interdependence information modifies behavior and thereby improves both objective and subjective outcomes at the group level. Improved performance is not a necessary outcome of increased mutual cooperation if, in combination, the other rounds included more mutual defection (yielding a point total of -2 for the two players) and fewer mixed outcomes (yielding a point total of 0 for the two players); likewise, satisfaction is not a necessary outcome of improved performance if players did not feel that the game was more fair (Blount, 1995). For this reason, we include these as separate hypotheses from those mentioned earlier.

\section{METHOD}

\section{Participants and procedure}

Participants $\left(N=240 ; 95\right.$ women, $145 \mathrm{men} ; M_{\mathrm{age}}=24.1$, $S D_{\text {age }}=5.51$ ) were recruited to a computer laboratory at Carnegie Mellon University and randomly paired with one another to play 200 unnumbered rounds of the IPD over the Internet. Players were not told the number of rounds in the game, and they were not numbered in the course of play. In each round, the two anonymous members of a pair (seated in different rooms without having met one another) chose simultaneously between buttons labeled Action A and Action B with payoffs as in Table 1. These payoffs were converted to incentive pay (one cent per point) beyond $\$ 10$ base pay. At the end of the game, participants rated their satisfaction with the outcome of the game on a five-point scale (1=Very dissatisfied, $2=$ Somewhat dissatisfied, $3=$ Neutral, $4=$ Somewhat satisfied, and $5=$ Very satisfied).

\section{Information conditions}

All participants saw their own action and payoff in each round. Thirty pairs (60 participants) were assigned to each of four conditions that determined the amount of information available about their interdependence. These conditions were modeled after the layers of the HSI outlined by Gonzalez and Martin (2011), although we simplify the labels here.

In the "No-Info" condition, individuals were not informed that they interacted with another player, so the selection of an 
action in each round was most likely perceived as an independent binary choice between two options with probabilistic payoffs. Participants may have realized that the probabilities of outcomes for each of the two actions were not static, as they in fact varied with the other player's actions, but this could more easily be attributed to a computerized process that shifted exogenously or in response to their own actions. In the "Min-Info" condition, players knew that their outcomes depended on the actions of another player and vice versa, yet still they did not know the other's specific actions and payoffs. With this information, individuals may have been able to speculate about the other's motivations, but it would remain difficult to empathize or coordinate unless both players correctly assumed the game was symmetrical and inferred the other's actions and payoffs. Next, pairs in the "Mid-Info" condition saw the actions and outcomes of the other player in each round, facilitating awareness that while defection would increase one's own payoffs and decrease the other player's payoffs in a given round, the other player would not tolerate this for long. Finally, in the "Max-Info" condition, in addition to seeing the other's actions and outcomes, players were shown the complete payoff matrix (as in Table 1) from the outset and throughout the repeated interaction. As such, both the immediate gain from defection and potential future gain from sustained mutual cooperation were made more explicit. The Appendix shows instructions to participants in each of the four conditions. Participants were not randomly assigned to conditions for logistical reasons, but we dispersed blocks of participants to different conditions throughout the course of the study to minimize confounds. The gender proportions were not significantly different across conditions (30\% women in No-Info, $50 \%$ women in Min-Info, $43 \%$ women in Mid-Info, and 35\% in Max-Info), $\chi^{2}(3, N=240)=5.91, p=.12$.

\section{Statistical tests}

We tested the overall effects of information condition in oneway ANOVAs and the differences between pairs of conditions in two-sample $t$-tests. Grouping observations into eight blocks of 25 rounds each, we assessed time trends within each condition using linear mixed effects models, clustering standard errors by either subject or pair, for analyses at the individual or pair level, respectively. For analyses of whether pair-wise cooperation mediated performance and satisfaction, we treated information as a continuous variable ( 0 = No-Info, 1 = Min-Info, 2 = Mid-Info, and 3= Max-Info $)$ in linear regressions in order to apply the Baron and Kenny procedure (1986) and Sobel tests.

\section{RESULTS}

\section{Individual cooperation}

Individual behavior varied considerably across the four conditions. As predicted, and as shown in Figure 2A, individuals exhibited a generally increasing average proportion of cooperation (equivalently, a decreasing proportion of defection) across conditions as information increased: $M=.21$ $(S D=.16)$ in the No-Info condition, $M=.21(S D=.16)$ in the Min-Info condition, $M=.31(S D=.29)$ in the Mid-Info condition, and $M=.43(S D=.35)$ in the Max-Info condition. Participants in the four conditions differed significantly in their proportion of cooperation, $F(3,236)=10.61, p<.001$, $f=.37$. This reflects greater rates of cooperation in the Max-Info condition compared with any of the other three, $t(118)>2.03, p<.05$, and in the Mid-Info condition compared with the two with less information, $t(118)>2.42$, $p<.02$. However, there was no difference between the MinInfo and No-Info conditions, $t(118)=-0.048, p=.96$.

Figure 2B illustrates that individual cooperation levels do not start out very different across conditions but gradually spread apart by later rounds as participants infer, correctly or not, how to optimize their outcomes. In fact, the proportion of cooperation was decreasing by block number (1-8, each consisting of 25 rounds) in the No-Info condition $(\beta=-0.023, t(419)=-5.94, p<.001)$ and in the Min-Info condition $(\beta=-0.022, t(419)=-6.62, p<.001)$, but increasing in the Mid-Info condition $(\beta=0.017, t(419)=4.03$,

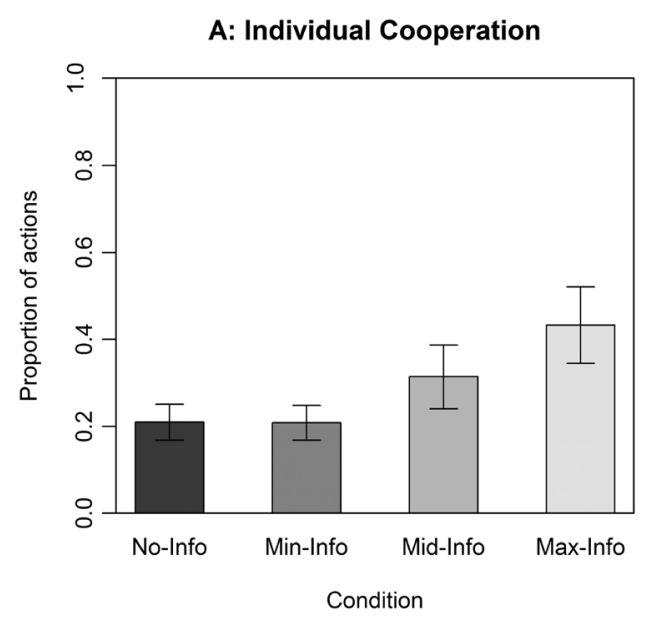

B: Time Course of Individual Cooperation

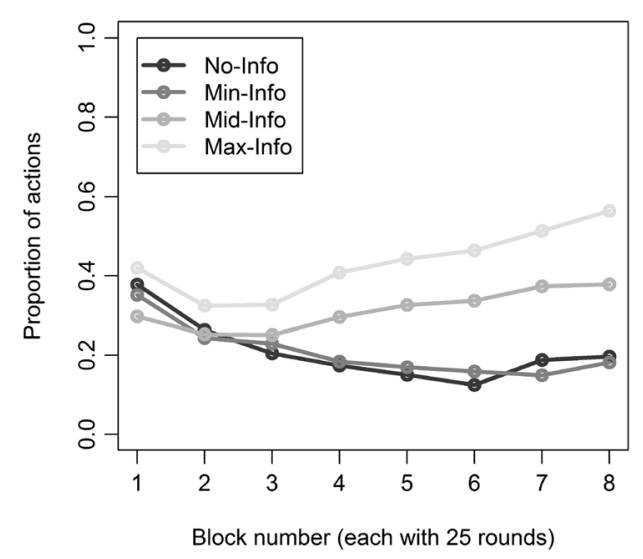

Figure 2. Proportion of individual cooperation in the IPD: (A) aggregated across 200 rounds with error bars showing 95\% confidence intervals, and (B) in each block of 25 rounds 
$p<.001)$ and in the Max-Info condition $(\beta=0.029, t(419)=6.26$, $p<.001)$.

\section{Mutual cooperation}

As shown in Figure 3A-F, outcomes for pairs of participants also varied across conditions. Like individual cooperation, the level of mutual cooperation was greater with more information: $M=.06(S D=.12)$ in the No-Info condition, $M=.06$
$(S D=.11)$ in the Min-Info condition, $M=.20(S D=.30)$ in the Mid-Info condition, and $M=.34(S D=.38)$ in the Max-Info condition (Figure 3A). There was no clear pattern for mixed outcomes (one player cooperating and the other defecting), which comprised the remaining pair outcomes (Figure 3B). Conversely, mutual defection was lower with more information: $M=.64(S D=.16)$ in the No-Info condition, $M=.61(S D=.18)$ in the Min-Info condition, $M=.57(S D=.27)$ in the Mid-Info condition,
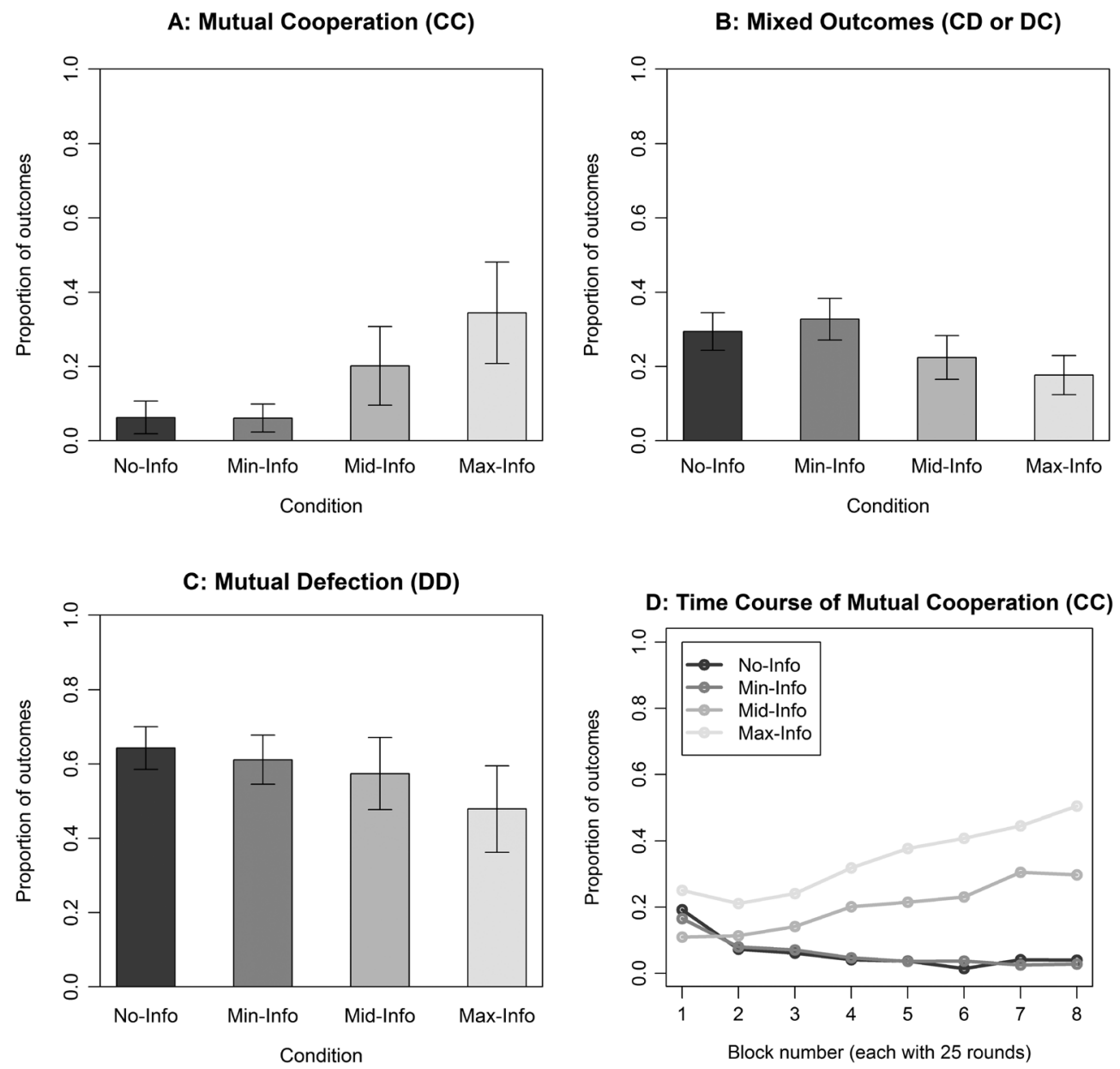

E: Time Course of Mixed Outcomes (CD or DC)
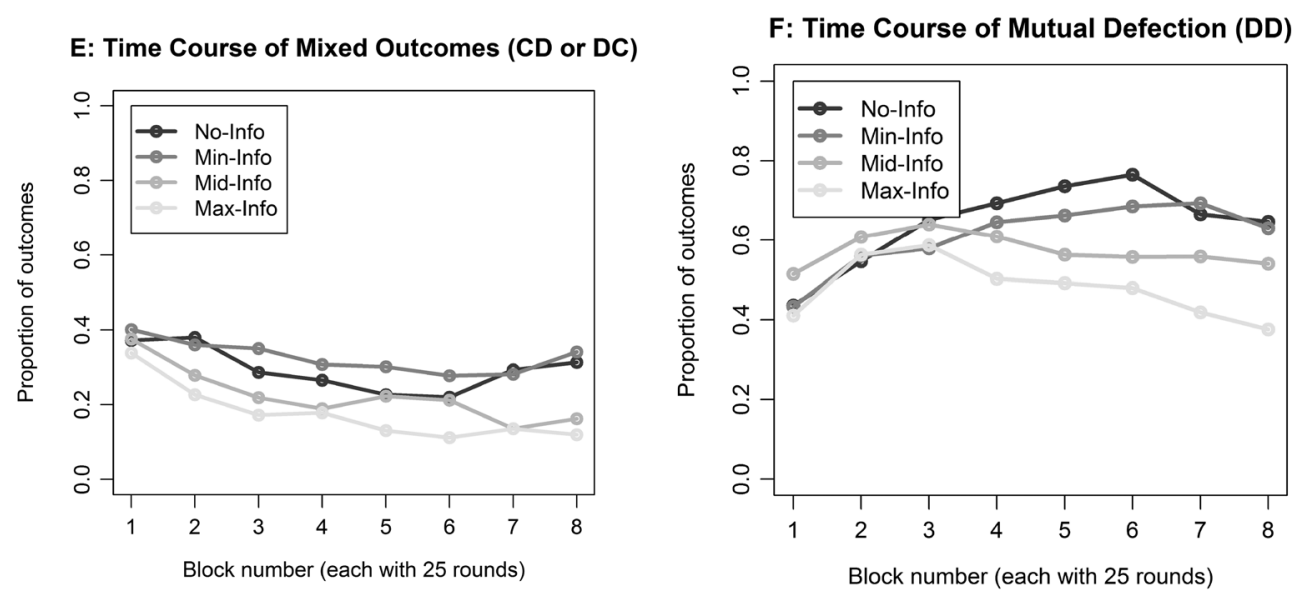

Figure 3. Proportion of pair outcomes in the IPD: (A) mutual cooperation, (B) mixed outcomes, and (C) mutual defection aggregated across 200 rounds, with error bars in each showing 95\% confidence intervals; time course of (D) mutual cooperation, (E) mixed outcomes, and (F) mutual defection in each block of 25 rounds 
and $M=.48(S D=.32)$ in the Max-Info condition (Figure 3C). Overall, information condition significantly affected mutual cooperation, $F(3,116)=8.44, p<.001, f=.47$. There were significant pair-wise differences in mutual cooperation between conditions, $t(58)<2.3, p<.03$, except no statistically significant difference between the Max-Info and Mid-Info conditions, $t(58)=1.62, p=.11$, and no difference between the Min-Info and No-Info conditions, $t(58)=-0.051, p=.96$.

While these pair-wise differences across conditions were relatively minor at the outset of the game, they became more prominent over time as participants responded to the available information. Mutual cooperation was decreasing by block at a similar rate in the No-Info condition $(\beta=-0.016$, $t(209)=-4.36, \quad p<.001)$ and the Min-Info condition $(\beta=-0.016, t(209)=-4.98, p<.001)$, but increasing in the Mid-Info condition $(\beta=0.030, t(209)=5.05, p<.001)$ and even more sharply in the Max-Info condition $(\beta=0.042$, $t(209)=6.56, p<.001$ ) (Figure 3D). Mixed pair-wise outcomes (CD and DC) were decreasing by block across all conditions as participants converged at extremes, suggesting that behavior did not remain purely random in the lower information conditions. However, the rate of decline in mixed outcomes was somewhat less in the No-Info condition $(\beta=-0.013, t(209)=-2.52, \quad p=.01)$ and the Min-Info condition $(\beta=-0.012, t(209)=-2.49, p=.01)$, as compared with the Mid-Info condition $(\beta=-0.026, t(209)=-7.41$, $p<.001)$ and the Max-Info condition $(\beta=-0.026$, $t(209)=-6.88, p<.001)$ (Figure $3 \mathrm{E})$. Mutual defection shows the reverse time trend from mutual cooperation, with increases by block in the No-Info condition $(\beta=0.029$, $t(209)=4.83, p<.001)$ and Min-Info condition $(\beta=0.028$, $t(209)=5.14, p<.001)$, but a non-significant decrease over time in the Mid-Info condition $(\beta=-0.004, t(209)=-0.71$, $p=.5)$ and significant decrease in the Max-Info condition $(\beta=-0.015, t(209)=-2.35, p=.02)$ (Figure 3F). Again, these time trends indicate that the effects of information accrue as decision makers integrate it with the seemingly self-reinforcing discovery of their own outcomes.

\section{Reciprocation and punishment}

The psychological processes underlying the aforementioned differences in behavior can be better isolated by understanding the proclivity of decision makers to mirror their counterpart's most recent decisions in accordance with a tit-for-tat strategy. Figure $4 \mathrm{~A}-\mathrm{C}$ displays this propensity contingent on whether one's counterpart cooperated or defected in the prior round. As predicted, we observed a general increase in reciprocal cooperation with more information: $M=.27$ $(S D=.24)$ in the No-Info condition, $M=.22(S D=.24)$ in

\section{A: Reciprocating Cooperation}

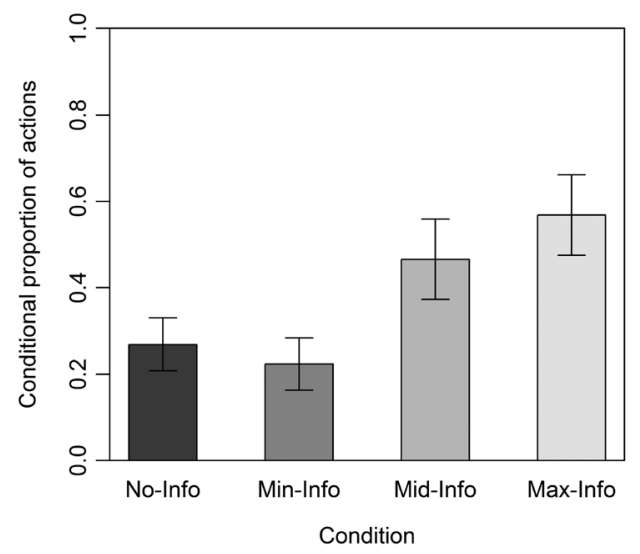

C: Time Course of Reciprocating Cooperation

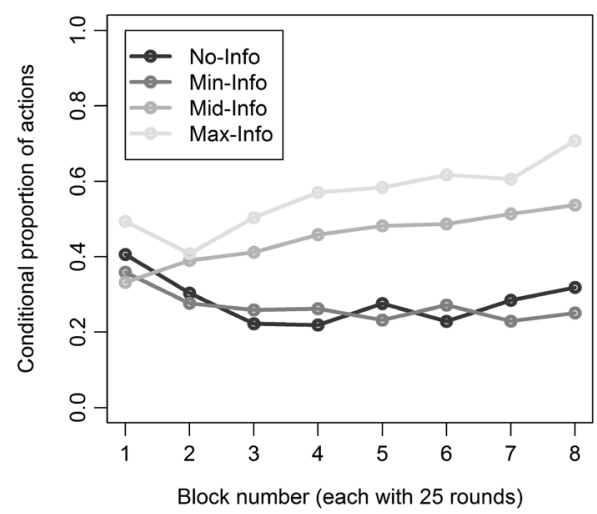

B: Punishing Defection

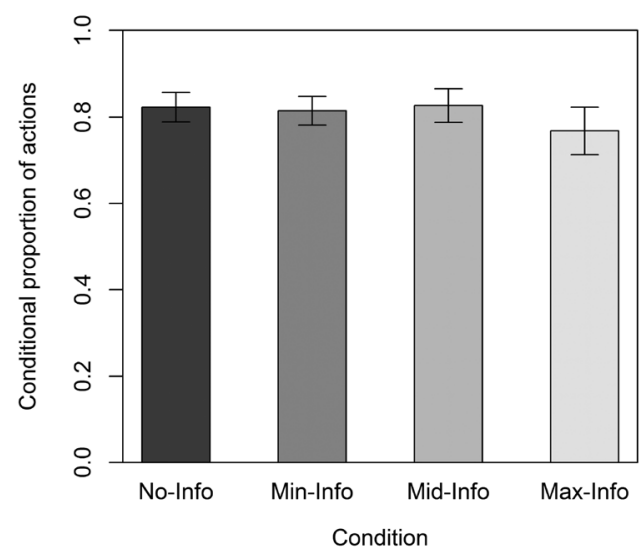

D: Time Course of Punishing Defection

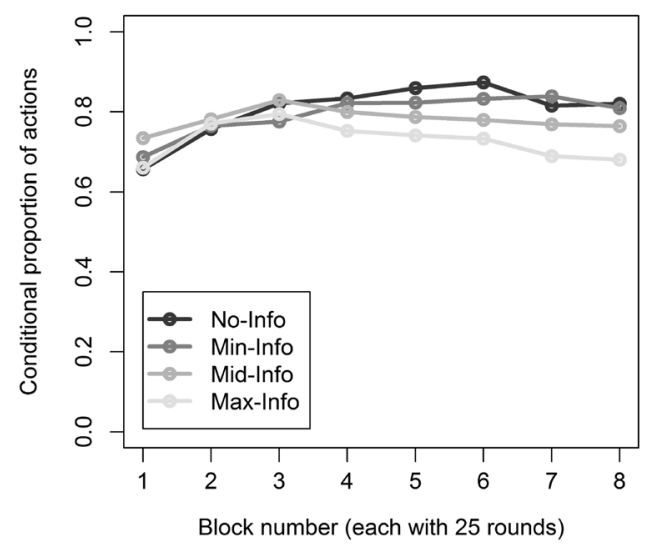

Figure 4. Use of a tit-for-tat strategy in the IPD: (A) reciprocation (proportion of cooperation contingent on a counterpart's cooperation in the prior round) and (B) punishment (proportion of defection contingent on a counterpart's defection in the prior round) aggregated across 200 rounds, with error bars in each showing $95 \%$ confidence intervals; (C) reciprocation and (D) punishment in each block of 25 rounds 
the Min-Info condition, $M=.47(S D=.37)$ in the Mid-Info condition, and $M=.57(S D=.37)$ in the Max-Info condition (Figure 4A). Reciprocal cooperation differed significantly by condition, $F(3,236)=16.4, p<.001, f=.46$, and there were significant differences between all pairs of conditions, $t(118)>3.47, p<.001$, except between the two conditions with the least information (No-Info and Mid-Info) and between the two conditions with the most information (Mid-Info and Max-Info), $t(118)<1.52, p>$.1.

Providing no additional evidence in favor of our prediction, however, punishment of defection was quite high across all conditions and showed no variation with information: $M=.82(S D=.14)$ in the No-Info condition, $M=.81$ $(S D=.13)$ in the Min-Info condition, $M=.83(S D=.15)$ in the Mid-Info condition, and $M=.77(S D=.22)$ in the MaxInfo condition (Figure 4B). There was no effect of condition on the whole, $F(3,236)=1.66, p=.2, f=.15$, and no pairs of conditions were different from one another, $t(118)<1.66$, $p>.1$, except for a marginal difference between the Mid-Info and Max-Info conditions, $t(118)=-1.70, p=.09$. In looking at the time trends in reciprocation and punishment, Figure 4C reveals the same time trend in reciprocal cooperation as for cooperation of the whole. Reciprocal cooperation was decreasing somewhat, although non-significantly, by time block in the No-Info condition $(\beta=-0.008, t(419)=-1.48$, $p=.14$ ) and decreasing more discernibly in the Min-Info condition $(\beta=-0.012, t(419)=-2.50, p=.01)$. By contrast, reciprocal cooperation was increasing across time blocks in the Mid-Info condition $(\beta=0.027, t(419)=5.46, p<.001)$ and in the Max-Info condition $(\beta=0.034, t(419)=6.96$, $p<.001)$.

Analyzing the time trends by block as visualized in Figure 4D, we see that reciprocating defection was increasing over time in the No-Info condition $(\beta=0.019, t(419)=5.41$, $p<.001)$ and Min-Info condition $(\beta=0.017, t(419)=4.90$, $p<.001)$, with a negligible decrease over time in both the Mid-Info condition $(\beta=-0.00018, t(419)=-0.061, p=.95)$ and the Max-Info condition $(\beta=-0.0055, t(419)=-1.52$, $p=.13)$. The time patterns were, thus, weaker on the whole for punishing defection than for reciprocating cooperation.

Overall, we can test the responsiveness of participants to their counterparts' most recent action by looking at whether participants were more likely to cooperate following a counterpart's cooperation than following a counterpart's defection. Even in the No-Info condition, there was a small average difference $M=0.092$ in the proportion of reciprocation following a counterpart's cooperation or defection, $t(59)=3.09, p=.003$. In the Min-Info condition, the average difference $M=0.039$ was even smaller and nonsignificant, $t(59)=1.49, p=.14$. However, as predicted, the difference was quite large in the Mid-Info condition, $M=0.29, t(59)=7.03, p<.001$, and in the Max-Info condition, $M=0.34, t(59)=8.50, p<.001$. By contrasting these differences in proportion of cooperation after a counterpart's cooperation or defection, there was an overall effect of condition, $F(3,236)=17.7, p<.001, f=.46$. In pair-wise comparisons, the differences were greater for both Max-Info and Mid-Info than for either Min-Info or No-Info, ts (118) $>3.92, p s<.001$, but similar in Min-Info and No-Info, $t(118)=-1.36, p=.2$, and in Max-Info and Mid-Info, $t(118)=0.76, p=.4$.

\section{Performance and satisfaction}

We next tested the hypothesis that interdependence information would improve game performance at the pair level. Performance was measured as the sum of points earned by the two players throughout the 200 rounds of IPD. Average point totals were negative in all conditions but less so with more information: $M=-232(S D=100)$ in the No-Info condition, $M=-220(S D=103)$ in the Min-Info condition, $M=-149 \quad(S D=218)$ in the Mid-Info condition, and $M=-53.7(S D=277)$ in the Max-Info condition. As a result, performance varied significantly across conditions, $F(3$, 116) $=5.57, p=.001, f=.38$. Performance was greater in the Max-Info condition than in the No-Info or Min-Info condition, $t(118)>3.08, p<.004$, and marginally greater in the Mid-Info than No-Info condition, $t(118)=1.90, p=.06$, but with no difference between the other three pairs, $t(118)<1.62, p>.1$.

As hypothesized, the effect of information on performance was mediated by mutual cooperation. Meeting the first Baron and Kenny (1986) criterion for mediation, we showed that information condition treated as a continuous variable $(0=$ No-Info, $1=$ Min-Info, $2=$ Mid-Info, and $3=$ Max-Info) positively predicted mutual cooperation, $\beta=0.099, t(118)=4.72, p<.001$. Achieving the second criterion, we showed that information condition positively predicted performance, $\beta=60.6, t(118)=3.91, \quad p<.001$. The third criterion for mediation requires that the effect of information become non-significant when mutual cooperation is included as another predictor of performance, which is what we found: the effect of mutual cooperation remained significant, $\beta=708, t(117)=34.3, p<.001$, while information became a marginally significant negative predictor of performance, $\beta=-9.21, t(117)=-1.80, p=.07$. A Sobel test confirmed that this reduction was significant, $Z=4.68$, $p<.001$, indicating that mutual cooperation mediated the relationship between our information manipulation and the cumulative performance of pairs. In fact, it appears that for pairs where more information failed to induce mutual cooperation, it actually led to lower joint payoffs due to more mutual defection (a sum of -2 points per round) and fewer mixed outcomes (a sum of 0 points per round).

Finally, we examined players' satisfaction with game outcomes. Satisfaction was computed as the sum of the two players' satisfaction ratings at the end of the game. In accordance with our hypothesis, average satisfaction values increased with information: $M=2.30(S D=1.78)$ in the NoInfo condition, $M=2.87(S D=1.98)$ in the Min-Info condition, $M=2.90(S D=2.07)$ in the Mid-Info condition, and $M=3.70$ $(S D=2.10)$ in the Max-Info condition. As a result, satisfaction varied marginally across conditions, $F(3,116)=2.51, p=.06$, $f=.25$. There was a significant difference between the two extreme conditions No-Info and Max-Info, $t(118)=2.78$, $p=.007$, but not between any other pairs of conditions, $t \mathrm{~s}(118)<1.58, p \mathrm{~s}>.1$. 
The effect of interdependence information on satisfaction was mediated by mutual cooperation, as predicted. Achieving the first Baron and Kenny (1986) criterion for mediation, we showed earlier that a pair's information condition predicted their proportion of mutual cooperation throughout the game. Second, information condition positively predicted joint satisfaction, $\beta=0.42, t(118)=2.62, p=.01$. Third, the effect of information became non-significant when mutual cooperation was included as another predictor of satisfaction: mutual cooperation remained significant, $\beta=3.80$, $t(117)=6.11, p<.001$, while information became nonsignificant, $\beta=0.048, t(117)=0.31, p=.8$. A Sobel test confirmed that this reduction was significant, $Z=3.74$, $p<.001$, indicating that mutual cooperation fully mediated the relationship between the information manipulation and pairs' satisfaction with game outcomes.

\section{DISCUSSION}

We tested the proposed effects of interdependence information using an IPD between anonymous players in the laboratory. Drawing upon Gonzalez and Martin's (2011) HSI, we designed four experimental conditions with incrementally increasing information provided to participants about their interdependence with one another. First, the absence of differences between the No-Info and Min-Info conditions is illuminating: it implies that mere knowledge of interdependence with another person is insufficient to promote reasoning about their motivations, despite the fact that the IPD is one of the simplest representations of a social dilemma, in that the two players' available actions and outcomes are symmetric. Compared with the No-Info and Min-Info conditions, though, cooperation rates were higher in the Mid-Info condition, suggesting that awareness of a counterpart's actions and outcomes improved inferences about their likely responses to one's own actions. Cooperation was even higher in the Max-Info condition where the visibility of the complete payoff matrix seemingly enabled players to be more forward-looking and proactive in recognizing the potential for shared benefits that can be achieved through sustained mutual cooperation. These differences across conditions were not evident from the start of the game, but rather cooperation was decreasing across rounds in the two lower information conditions and increasing across rounds in the two higher information conditions. It appears that participants with less descriptive information only learned that defection was optimal in any given round, whereas those with more information learned additionally that their own choices influenced the "probabilities" of good outcomes in subsequent rounds.

To examine the psychological processes by which interdependence information fostered cooperation, we measured propensities for reciprocation (proportion of cooperation contingent on a counterpart's prior cooperation) and punishment (proportion of defection contingent on a counterpart's prior defection), together comprising a tit-for-tat which is a strategy shown to be effective in the IPD (Axelrod, 1980). The results revealed that increasing cooperation with information was driven in part by players' greater willingness to reciprocate prior cooperation by the other player. However, participants exhibited nearly identical levels of punishing defection across conditions, indicating that any differences in either forgiveness or hostility due to information about another's defection may have counteracted one another. Comparing Figures 2 and 4, we note several important points: in each of the four conditions, overall rates of cooperation (not contingent on a partner's prior action) were lower than rates of reciprocal cooperation (contingent on a partner's prior cooperation), and overall rates of defection (not contingent on a partner's prior action) were lower than rates of punishing defection (contingent on a partner's prior defection); however, the differences between non-contingent and contingent cooperation, and between non-contingent and contingent and defection, widened for conditions with more information. Individuals possessing more interdependence information were not simply reacting to the tangible benefits or costs that would accrue to themselves but also enforcing social norms of reward and retribution because of the other player.

A key implication of this experiment is the generally positive impact of information on joint performance and satisfaction. It appears that when information about the IPD incentive structure was common knowledge, members of a pair were able to recognize the potential for mutual gain and then act upon this foresight by simultaneously taking the risk of cooperation. Pairs that achieved coordinated cooperation capitalized via higher combined payoffs and reported higher satisfaction with the game outcome. We speculate-but it requires more precise measurement in further research-that additional information may have some robust favorable effects across other types of interactions, too. For one, information may diminish the "fundamental attribution error" whereby individuals assume ill intentions of others who behave in an antisocial manner but ascribe their own antisocial behavior to situational factors (Jones \& Harris, 1967; Ross, 1977). In addition, information may reduce focus on the immediate gains of acting selfishly (e.g., defection in the IPD) and on loss aversion with respect to the risk of a poor relative outcome (e.g., from unilateral cooperation against a defecting counterpart in the IPD) (Kahneman \& Tversky, 1979). More generally, information may enhance "theory of mind," or imputation mental states, for others (Premack \& Woodruff, 1978), particularly when one understands that a counterpart faces a similar predicament to one's own.

\section{CONCLUSIONS}

There are frequent instances in our lives where we fail to fully comprehend our interdependence with others and specifically our ability to advance individual and collective well-being through interpersonal influence. The IPD is a useful starting point for the study of interdependence information because it captures pervasive tensions between local and global outcomes, and between short-term and long-term considerations, that characterize a variety of social interactions including arms races (Axelrod, 1980), international trade (Conybeare, 1984), and entrepreneur-venture 
capitalist relationships (Cable \& Shane, 1997). In each of these social dilemmas, our experimental results predict that incremental knowledge of interdependence will guide decision makers toward cooperation, perpetuated by positive responses to the prior cooperation of others but not necessarily by a reduction in punishment of defection by others. This strategic shift may occur at the level of those in power, such as public officials or business managers, or it might also bolster general public support for more cooperative policies like nuclear disarmament.

Consider the environmental "public goods" problem as a multi-player game of IPD between individuals, households, governments, and corporations. Most actors have only a basic notion of their interdependence with others, which, according to our laboratory findings, is an insufficient condition for cooperative behavior (in this case, conservation of natural resources). However, if decision makers were able to observe one another's actions and outcomes over time, accountability would rise along with the prospect of reciprocation. The greatest social benefits would accrue if actors were given more explicit descriptive information about the interaction structure, specifying the causality between sets of actions and joint outcomes. In the environmental dilemma, it is difficult to reach scientific consensus on the content of a numeric payoff matrix, yet our results indicate that it may behoove actors to make their subjective costs and benefits more transparent to one another even though this presents the danger of exploitation. One important direction for future research is to investigate how the voluntary sharing of truthful information can be encouraged despite the risk it poses. Another is the applicability of our findings to a wider variety of social dilemmas, including those with asymmetric information, payoffs, or power dynamics.

\section{APPENDIX INSTRUCTIONS TO PARTICIPANTS}

\section{NO-INFO CONDITION}

In this experiment, you will play a computer game. This game consists of a series of rounds in which you will make decisions that affect your payoffs. In each round, you can choose either Action A or Action B, and your payoffs will depend on your action as well as other factors.

In addition to other earnings, your cumulative point total from this series of rounds will be converted to money at the end of the experiment, with each point worth 1 cent.

\section{MIN-INFO CONDITION}

You have been randomly matched with an anonymous opponent in the other lab to play an interactive game. This game consists of a series of rounds in which you and your opponent will make simultaneous decisions that affect each of your payoffs. In each round, each of you can choose either Action A or Action B, and both of your payoffs will depend on your own action as well as your opponent's action. However, neither of you will see what action the other took or what the other's payoffs were. Each of you will see only your own action and your own payoff.

In addition to other earnings, your cumulative point total from this series of rounds will be converted to money at the end of the experiment, with each point worth 1 cent.

\section{MID-INFO CONDITION}

You have been randomly matched with an anonymous opponent in the other lab to play an interactive game. This game consists of a series of rounds in which you and your opponent will make simultaneous decisions that affect each of your payoffs. In each round, each of you can choose either Action A or Action B, which will be revealed at the same time, and both of your payoffs will depend on your own action as well as your opponent's action. You will only learn these payoffs as you play the game.

In addition to other earnings, your cumulative point total from this series of rounds will be converted to money at the end of the experiment, with each point worth 1 cent.

\section{MAX-INFO CONDITION}

You have been randomly matched with an anonymous opponent in the other lab to play an interactive game. This game consists of a series of rounds in which you and your opponent will make simultaneous decisions that affect each of your payoffs. In each round, each of you can choose either Action A or Action B, which will be revealed at the same time, and both of your payoffs will depend on your own action as well as your opponent's action, as shown in the following table:

Opponent's action

A

B

\begin{tabular}{llll}
\hline Your Action & A & You get -1 point, opponent gets -1 point & You get 10 points, opponent gets -10 points \\
& B & You get -10 points, opponent gets 10 points & You get 1 point, opponent gets 1 point \\
\hline
\end{tabular}

In addition to other earnings, your cumulative point total from this series of rounds will be converted to money at the end of the experiment, with each point worth 1 cent. 


\section{ACKNOWLEDGEMENTS}

This research was supported by the Defense Threat Reduction Agency grant HDTRA1-09-1-0053 to Cleotilde Gonzalez and Christian Lebiere.

\section{REFERENCES}

Axelrod, R. (1980). Effective choice in the prisoner's dilemma. Journal of Conflict Resolution, 24(1), 3-25. DOI: 10.1177/ 002200278002400101

Axelrod, R. (1984). The evolution of cooperation. New York: Basic Books.

Axelrod, R., \& Hamilton, W. D. (1981). The evolution of cooperation. Science, 211(4489), 1390-1396. DOI: 10.1126/science.7466396

Baker, F., \& Rachlin, H. (2001). Probability of reciprocation in repeated prisoner's dilemma games. Journal of Behavioral Decision Making, 14, 51-67. DOI: 10.1002/1099-0771(200101) $14: 1<51::$ AID-BDM365>3.0.CO;2-K

Bandura, A. (1982). Self-efficacy mechanism in human agency. American Psychologist, 37(2), 122-147. DOI: 10.1037/0003-066X.37.2.122

Baron, R. M., \& Kenny, D. A. (1986). The moderator-mediator variable distinction in social psychological research: Conceptual, strategic, and statistical considerations. Journal of Personality and Social Psychology, 51(6), 1173-1182. DOI: 10.1037/ 0022-3514.51.6.1173

Barron, G., \& Erev, I. (2003). Small feedback-based decisions and their limited correspondence to description-based decisions. Journal of Behavioral Decision Making, 16, 215-233. DOI: 10.1002/bdm.443

Blount, S. (1995). When social outcomes aren't fair: The effect of causal attributions on preferences. Organizational Behavior and Human Decision Processes, 63(2), 131-144. DOI: 10.1006/obhd.1995.1068

Cable, D. M., \& Shane, S. (1997). A prisoner's dilemma approach to entrepreneur-venture capitalist relationships. Academy of Management Review, 22(1), 142-176.

Camerer, C. F., \& Fehr, E. (2006). When does "Economic Man" dominate social behavior? Science, 311(5757), 47-52. DOI: $10.1126 /$ science. 1110600

Camerer, C., (2003). Behavioral game theory: Experiments in strategic interaction. Princeton University Press.

Charness, G., \& Rabin, M. (2002). Understanding social preferences with simple tests. Quarterly Journal of Economics, 117 (3), 817-869. DOI: 10.1162/003355302760193904

Cialdini, R. B., Reno, R. R., \& Kallgren, C. A. (1990). A focus theory of normative conduct: Recycling the concept of norms to reduce littering in public places. Journal of Personality and Social Psychology, 58(6): 1015-1026.

Clark, K., \& Sefton, M. (2001). The sequential prisoner's dilemma: Evidence on reciprocation. The Economic Journal, 111(468), 51-68. DOI: 10.1111/1468-0297.00588

Conybeare, J. A. C. (1984). Public goods, prisoners' dilemmas and the international political economy. International Studies Quarterly, 28(1), 5-22.

Cox, J. C. (2004). How to identify trust and reciprocity. Games and Economic Behavior, 46(2), 260-281. DOI: 10.1016/S0899-8256 (03)00119-2

Fantino, E., \& Navarro, A. (2012). Description-experience gaps: Assessments in other choice paradigms. Journal of Behavioral Decision Making, 25, 303-314. DOI: 10.1002/bdm.737

Festinger, L. (1954). A theory of social comparison processes. Human Relations, 7, 117-140. DOI: 10.1177/001872675400700202

Festinger, L. (1957). A theory of cognitive dissonance. Stanford, CA: Stanford University Press.

Gallagher, H. L., Jack, A. I., Roepstorff, A., \& Frith, C. D. (2002). Imaging the intentional stance in a competitive game. Neurolmage, 16, 814-821. DOI:10.1006/nimg.2002.1117
Gonzalez, C., \& Martin, J. M. (2011). Scaling up instance-based learning theory to account for social interactions. Negotiation and Conflict Management Research, 4(2), 110-128. DOI: 10.1111/j.1750-4716.2011.00075.x

Hadar, L., \& Fox, C. R. (2009). Information asymmetry in decision from description versus decision from experience. Judgment and Decision Making, 4(4), 317-325.

Halevy, N., Sagiv, L., Roccas, S., \& Bornstein, G. (2006). Perceiving intergroup conflict: From game models to mental templates. Personality and Social Psychology Bulletin, 32, 1674-1689. DOI: $10.1177 / 0146167206291947$

Hau, R., Pleskac, T. J., \& Hertwig, R. (2009). Decisions from experience and statistical probabilities: Why they trigger different choices than a priori probabilities. Journal of Behavioral Decision Making, 23(1), 48-68. DOI: 10.1002/bdm.665

Hau, R., Pleskac, T. J., Kiefer, J., \& Hertwig, R. (2008). The description-experience gap in risky choice: The role of sample size and experienced probabilities. Journal of Behavioral Decision Making, 21(5), 493-518. DOI: 10.1002/bdm.598

Hertwig, R., Barron, G., Weber, E. U., \& Erev, I. (2004). Decisions from experience and the effect of rare events in risky choice. Psychological Science, 15(8), 534-539. DOI: 10.1111/j.09567976.2004.00715.x

Hwang, P., \& Burgers, W. P. (1997). Properties of trust: An analytical view. Organizational Behavior and Human Decision Processes, 69(1), 67-73. DOI: 10.1006/obhd.1996.2673

Jones, E. E., \& Harris, V. A. (1967). The attribution of attitudes. Journal of Experimental Social Psychology, 3(1), 1-24. DOI: 10.1016/0022-1031(67)90034-0

Juvina, I., Saleem, M., Martin, J. M., Gonzalez, C., \& Lebiere, C. (2013). Reciprocal trust mediates deep transfer of learning between games of strategic interaction. Organizational Behavior and Human Decision Processes, 120(2), 206-215. DOI: http:// www.sciencedirect.com/science/article/pii/S0749597812001215

Kahneman, D., \& Tversky, A. (1979). Prospect theory: An analysis of decision under risk. Econometrica, 47(2), 263-291. DOI: $10.2307 / 1914185$

Kelley, H. H., \& Thibaut, J. W. (1978). Interpersonal relations: A theory of interdependence. New York, NY: Wiley.

Kreps, D. M., \& Wilson, R. (1982). Reputation and imperfect information. Journal of Economic Theory, 27, 253-279. DOI: 10.1016/0022-0531(82)90030-8

Lejarraga, T. \& Gonzalez, C. (2011). Effects of feedback and complexity on repeated decisions from description. Organizational Behavior and Human Decision Processes, 116, 286-295. DOI: 10.1016/j. obhdp.2011.05.001

Ludvig, E. A., \& Spetch, M. L. (2011). Of black swans and tossed coins: Is the description-experience gap in risky choice limited to rare events? PLoS ONE, 6(6), e20262. DOI:10.1371/journal. pone. 0020262

McCabe, K., Houser, D., Ryan, L., Smith, V., \& Trouard, T. (2001). A functional imaging study of cooperation in twoperson reciprocal exchange. Proceedings of the National Academy of Sciences, 98(20), 11832-11835. DOI: 10.1073/ pnas. 211415698

Moore, D. A., Kurtzberg, T. R., Thompson, L. L., \& Morris, M. W. (1999). Long and short routes to success in electronically mediated negotiations: Group affiliations and good vibrations. Organizational Behavior and Human Decision Processes, 77(1), 22-43. DOI: 10.1006/obhd.1998.2814

Nisbett, R. E., \& Ross, L. (1980). Human inference: Strategies and shortcomings of social judgment. Englewood Cliffs, NJ: PrenticeHall.

Pillutla, M. M., \& Chen, X. (1999). Social norms and cooperation in social dilemmas: The effects of context and feedback. Organizational Behavior and Human Decision Processes, 78(2), 81-103. DOI: 10.1006/obhd.1999.2825

Premack, D., \& Woodruff, G. (1978). Does the chimpanzee have a theory of mind? Behavioral and Brain Sciences, 1(4), 515-526. DOI: $10.1017 / \mathrm{S} 0140525 \mathrm{X} 00076512$ 
Rabin, M. (1993). Incorporating fairness into game theory and economics. American Economic Review, 83, 1281-1302. DOI: 10.2307/2117561

Rakow, T., Demes, K. A., \& Newell, B. R. (2008). Biased samples not mode of presentation: Re-examining the apparent underweighting of rare events in experience-based choice. Organizational Behavior and Human Decision Processes, 106, 168-179. DOI: 10.1016/j.obhdp.2008.02.001

Rapoport, A., \& Chammah, A. M. (1965). Prisoner's dilemma: A study in conflict and cooperation. Ann Arbor, MI: University of Michigan Press.

Rilling, J. K., Goldsmith, D. R., Glenn, A. L., Jairam, M. R., Elfenbein, H. A., Dagenais, J. E., Murdocka, C. D., \& Pagnoni, G. (2008). The neural correlates of the affective response to unreciprocated cooperation. Neuropsychologia, 46(5), 1256-1266. DOI:10.1016/j.neuropsychologia.2007.11.033

Rilling, J. K., Sanfey, A. G., Aronson, J. A., Nystrom, L. E., Cohen, J. D. (2004). The neural correlates of theory of mind within interpersonal interactions. NeuroImage, 22, 1694-1703. DOI: 10.1016/j.neuroimage.2004.04.015

Ross, L. (1977). The intuitive psychologist and his shortcomings: Distortions in the attribution process. In L. Berkowitz (Ed.), Advances in experimental social psychology (Vol. 10, pp. 173-220). New York: Academic Press.

Roth, A. E., \& Murnighan, J. K. (1978). Equilibrium behavior and repeated play of the prisoner's dilemma. Journal of Mathematical Psychology, 17, 189-198. DOI: 10.1016/0022-2496(78)90030-5

Rusbult, C. E., \& Van Lange, P. A. M. (2003). Interdependence, interaction, and relationships. Annual Review of Psychology, 54, 351-375. DOI: 10.1146/annurev.psych.54.101601.145059

Tetlock, P. E. (1992). The impact of accountability on judgment and choice: Toward a social contingency model. In M. Zanna (Ed.), Advances in experimental social psychology, 25, 331-376. New York, NY: Academic Press.

Tinsley, C. H., O’Connor, K. M., \& Sullivan, B. A. (2002). Tough guys finish last: The perils of a distributive reputation. Organizational Behavior and Human Decision Processes, 88(2), 621-64. DOI: 10.1016/S0749-5978(02)00005-5

Ungemach, C., Chater, N., \& Stewart, N. (2009). Are probabilities overweighted or underweighted when rare outcomes are experienced (rarely)? Psychological Science, 20(4), 473-479. DOI: 10.1111/j.1467-9280.2009.02319.x

Yechiam, E., Barron, G., \& Erev, I. (2005). The role of personal experience in contributing to different patterns of response to rare terrorist attacks. Journal of Conflict Resolution, 49, 430-439. DOI: $10.1177 / 0022002704270847$

Yechiam, E., \& Busemeyer, J. R. (2006). The effect of foregone payoffs on underweighting small probability events. Journal of Behavioral Decision Making, 19(1), 1-16. DOI: 10.1002/bdm.509
Yechiam, E., Druyan, M., \& Ert, E. (2008). Observing others' behavior and risk taking in decisions from experience. Judgment and Decision Making, 3(7), 493-500.

\section{Authors' biographies:}

Jolie Martin is a Quantitative User Experience Researcher at Google Inc. She uses a variety of data-driven and computational modeling approaches to investigate human information processing, judgment and decision making, and social interaction. Applications of her work extend to consumer choice and behavioral economics.

Cleotilde (Coty) Gonzalez is an Associate Research Professor in the Department of Social and Decision Sciences at Carnegie Mellon University. She is also the Founding Director of the Dynamic Decision Making Laboratory. Her interdisciplinary research program focuses on the study of human decision making in dynamic and complex environments.

Ion Juvina is an Assistant Professor in the Department of Psychology at Wright State University. His research focuses on high-level cognitive processes such as strategic thinking and executive control of cognition and emotion, combining empirical research, and computational cognitive modeling.

Christian Lebiere is a Research Faculty in the Psychology Department at Carnegie Mellon University. Since 1991, he has worked on the development of the ACT-R cognitive architecture. His research interests include cognitive architectures and applications to cognitive psychology, artificial intelligence, human-computer interaction, decision making, intelligent agents, cognitive robotics, and neuromorphic engineering.

Authors' addresses:

Jolie M. Martin, Social and Decision Sciences, Carnegie Mellon University, Pittsburgh, PA, USA.

Cleotilde Gonzalez, Social and Decision Sciences, Carnegie Mellon University, Pittsburgh, PA, USA.

Ion Juvina, Psychology, Wright State University, Dayton, OH, USA.

Christian Lebiere, Psychology, Carnegie Mellon University, Pittsburgh, PA, USA. 